\title{
Determination of cavitation load spectra - Part 1: Static finite element approach
}

\author{
Samir Chandra Roy ${ }^{a, k}$, Jean-Pierre Franc ${ }^{b}$, Christian Pellone ${ }^{b}$, Marc Fivel ${ }^{a}$ \\ a Science and Engineering of Materials and Processes (SIMaP-GPM2), University Grenoble Alpes / CNRS, F-38000 Grenoble, France \\ Laboratory of Geophysical and Industrial Flows (LEGI), University Grenoble Alpes / CNRS, F-38000 Grenoble, France
}

\begin{abstract}
Numerical prediction of cavitation damage strongly relies on the determination of the loading conditions applied to the wall. In this paper, an inverse method is proposed to identify the pressure field that could generate individual pits as observed experimentally on eroded samples of Aluminum alloy 7075-T651. The pits are defined by the diameter and depth of the imprints. Assuming each pit was generated by a single bubble collapse, the pressure load is defined by two parameters, the peak pressure $\left(\sigma_{H}\right)$ and its radial extent $\left(r_{H}\right)$. Two methods are proposed based on finite element modeling. The first one uses analytical expression of the unknown parameters built from a parametric simulation campaign. The second one is based on an optimization loop of the finite element simulations to best fit the experimental measures for a given error limit. Both methods give access to the load distributions relevant to the flow aggressiveness of the cavitation test.
\end{abstract}

Keywords: Cavitation pitting; Pit dimensions; Hydrodynamic impact parameters; Analytical inverse method; Numerical inverse method

\section{$1 \quad$ Introduction}

It is well known that the repeated collapse of cavitation bubbles may erode solid walls [1-3]. The collapse of a cavitation bubble is generally associated with the formation of a micro-jet and/or shock waves, which impact the nearby solid surface resulting in a cavitation pit, if the load is high enough to exceed the local yield strength of the material. In order to predict the erosion damage including the long-term damage and mass loss, it is essential to know the loading conditions generated by bubble collapses and analyze the response of the material to these loads.

The determination of the loading conditions due to the combined or solo effect of micro-jet and shock waves during cavitation bubble collapse is a major issue in cavitating flows. Numerical approaches may be used to compute the pressure pulses due to the collapse of a single bubble or bubble clusters that may develop in real flows such as the flow around a cavitating foil or in a cavitating hydraulic device (see e.g. [4-6]). The difficulties in such approach arise from the complex fluid-structure interaction and also from the large number of parameters involved on the fluid side such as bubble content, bubble size, distance to the wall, pressure history to which the bubble is subjected, potential interactions between bubbles, etc.

Pressure pulses may also be measured in cavitation facilities. One option is to use pressure transducers flush mounted in the region of bubble collapses. The pressure signal generally shows successive pulses of various amplitudes caused by bubble collapses [7-9]. This method allows determination of impact loads in force units (typically in Newton) but the determination of the pressure or stress amplitude (in MPa) is difficult because the loaded surface area is unknown and usually much smaller than the transducer sensitive surface. Moreover, conventional pressure transducers, because of

\footnotetext{
${ }^{*}$ Corresponding author:

E-mail address: roysam.nita@gmail.com or samir-chandra.roy@simap.grenoble-inp.fr (S. C. Roy)
} 
their limited natural frequency, may not capture accurately the cavitation pressure pulses whose rise time and duration are quite small. Finally, the impact could plastically deform or even damage the transducer leading to faulty responses. In order to overcome these measurement difficulties, another option may be used. It consists in using the material itself as a transducer. The measuring technique is based on pitting tests as introduced by [10, 11]. The idea behind pitting tests is that each pit is the signature of a single bubble collapse. Then, it can reasonably be expected that the loading conditions be derived from the geometry of the pit and the material properties.

\section{Nomenclature}

$r \quad$ Radial direction for an axisymmetric element

$z \quad$ Longitudinal direction for an axisymmetric element

$R \quad$ Maximum radial size of the simulated volume

$H \quad$ Vertical size along $\mathrm{z}$ axis of the simulated volume

$\sigma_{H} \quad$ Peak pressure of hydrodynamic impact

$r_{H} \quad$ Radial extent of hydrodynamic impact

$d_{H} \quad$ Diameter of hydrodynamic impact

$h_{P} \quad$ Pit depth

$d_{P} \quad$ Pit diameter at $50 \%$ of pit depth

$r_{P} \quad$ Pit radius at $50 \%$ of pit depth

$h \quad$ Depth (Eq. (6))

$d \quad$ Diameter (Eq. (6))

$h_{\max } \quad$ Maximum pit depth (Eq. (6))

$d_{\max } \quad$ Maximum pit diameter (Eq. (6))

$P_{0} \quad$ Maximum Hertz pressure

$r_{c} \quad$ Hertzian contact radius

$T \quad$ Shear stress along depth, $\mathrm{z}$ on the axis of symmetry

$\sigma \quad$ True stress

$\varepsilon \quad$ True strain

$\dot{\varepsilon} \quad$ Strain rate

$\sigma_{y} \quad$ Elastic limit

$m_{y} \quad$ Parameter of the Ramberg-Osgood constitutive equation

$K_{y} \quad$ Parameter of the Ramberg-Osgood constitutive equation

$\varepsilon_{e} \quad$ Elastic strain

$\varepsilon_{P} \quad$ Plastic strain

$\sigma_{u} \quad$ Ultimate stress

$\varepsilon_{u} \quad$ Ultimate strain

E Young's modulus

$v \quad$ Poisson's ratio

$k \quad$ Material constant (Eq. (3)))

$\sigma^{*}, \beta \quad$ Material constants ( Eq. (4))

$\sigma_{e q} \quad$ Von Mises equivalent stress

$N \quad$ Cumulative pitting rate

$h_{c} \quad$ Cut-off depth (Eq. (7))

$\sigma_{H_{\min }} \quad$ Minimum value of peak pressure require to form a pit (Eq. (7))

Such a technique has been used by $[12,13]$. The authors have taken advantage of the similarity between a cavitation erosion pit and a spherical nanoindentation to estimate the amplitude of the pressure pulse responsible for a cavitation pit. The method is based on the use of Tabor's equation [14] that makes it possible to estimate the mean strain associated to a plastic deformation of given depth and diameter. It is easy to deduce the stress from the estimated strain using the stress-strain relationship of the material.

In the present work, another technique is investigated for deriving the loading conditions from pitting tests conducted during the incubation period. It is based on finite element (FE) computations of the response of the material to a representative pressure pulse. The pressure pulse considered here has a Gaussian shape and is defined by two parameters, namely its maximum amplitude and radial extent. The Gaussian shape profile used for the FE simulations is 
found to produce non-dimensional pit shapes that are close to that experimentally observed, as discussed in Section 4. An inverse technique is proposed to derive these two parameters from the depth and diameter of the pit, both deduced from an appropriate analysis of the pitted surface.

This kind of approach, combining FE simulations and pitting test is relatively new and the authors have noticed only few publications. To our knowledge, such an inverse technique has only been used by Phol et al. [15] using a different bell-shape pressure profile into the framework of static FE (no time dependencies) analysis of material response using 2D axisymmetric modeling. We are proposing here a simple and fast technique based on interpolation that optimizes the pressure parameters for given error limits in pit dimensions. Moreover, we show that a given pit shape could be optimized with a unique set of parameters for the assumed pressure profile. Note that in order to simplify the problem, Phol et al. [15] have ignored dynamic effect that include inertia and strain rate sensitivity of the material, as we are doing in this current Part 1 paper.

The event of cavitation hydrodynamic impact is very dynamic in nature since the impact duration is very short, in the order of a microsecond, as observed experimentally and/or by computational fluid dynamics (CFD) simulation of cavitation bubble collapse [5, 16, 17, 18, 19]. In a companion paper (Part 2) [20], we have performed dynamic explicit FE analysis of the cavitation impact using similar Gaussian pressure field with a temporal evolution of Gaussian type. By decoupling the effect of inertia and strain rate sensitivity into the simulation, it was found that for impact duration of $1 \mu \mathrm{s}$ or more the inertial effect becomes insignificant and, static and dynamic explicit FE analyses yield the same solution in terms of resulted pit dimensions. Similar observation was reported by Choi et al. in [19]. However, strain rate effect at such high rate of loading in cavitation pitting cannot be avoided if the material is strain rate sensitive. For more details see [20], where it is shown that for duplex stainless steel (A-2205) which has high strain rate sensitivity, although inertial effect is negligible for impact durations as small as $1 \mu \mathrm{s}$, the dynamic effect associated with strain rate sensitivity is unavoidable till $10^{5} \mu \mathrm{s}$ (or $0.1 \mathrm{~s}$ ) of impact duration; thus care should be taken as described in [20]. In order to avoid the effects of strain rate in our modeling approach, the current study is conducted on Aluminum alloy 7075-T651 (A1-7075) which has very weak strain rate sensitivity [21] so that a static approach appears fully appropriate for this particular alloy even for impact durations as short as $1 \mu \mathrm{s}$.

The strain rate sensitivity coefficient of Al-7075 was estimated by using the well-known Johnson-Cook plasticity model, for which compression and Split Hopkinson Pressure Bar (SHPB) tests were done for strain rates ranging from 0.001 to $\sim 2000 \mathrm{~s}^{-1}$, details of which can be found in [21]. A value $C=0.0068$ for the strain rate parameter was found which demonstrates negligible strain rate sensitivity.

Although in the current study, static analysis was adopted for the inverse FE technique, it is shown in Part 2 [20] that this inverse technique could be transposed to dynamic explicit analysis as well and then applied to strain rate sensitive materials by considering the complete dynamic behavior including inertia and strain rate sensitivity. The question of impact duration corresponding to each cavitation pit remains however unsolved. The authors $[5,16,17]$ have both experimentally and numerically determined the impact durations corresponding to cavitation bubble collapses. However, the geometry and the cavitation conditions considered in these studies are significantly different from the conditions considered in the present paper, so that the transposition of these data to the present case is not straightforward. To our knowledge, the impact duration cannot be determined from the sole pit shape that represents the final plastic deformation and time sensitive transducers are required to provide details on the time evolution of the impact load including its duration. In the case, the impact duration is unknown; it may be difficult to use a fully dynamic approach. For strain rate sensitive material, one option could be to use a static approach and extrapolate the 
material properties to a high strain rate that would correspond to the typical strain rates involved in cavitation impacts (for more details see [21]).

The inverse FE technique has been implemented in [21] for different cavitation flow conditions and impact loads were estimated by using three different materials as sensors. Very interestingly, statistical analyses of the estimated impact loads show a material independent response for a given flow condition. This is very encouraging and proves the reliability of the estimated impact loads.

The FE computation technique is presented and validated against the Hertzian elastic contact theory in Section 2. Typical results of FE pitting calculations are presented and discussed in Section 3 for the aluminum alloy Al-7075 and an approximate analytical inverse technique is presented. Section 4 is devoted to the technique of analysis of pitting tests for the determination of pit depth and diameter. In Section 5, the principle of the inverse FE technique termed here as numerical inverse method is presented. Typical results of the inverse technique are given and discussed in Section 6 , which provide a kind of validation to the estimated impact loads.

\section{Numerical method}

\subsection{Model considerations and verification}

In the current study, as well as in [15] most of the experimental pit shapes are found to be nearly axially symmetric. Such a pit shape is shown in Fig. 12. So the effective pressure field could also be assumed symmetric and hence a semiinfinite 2D axisymmetric model as shown in Fig. 1 is used for the current study. Although static FE analyses were conducted for simplicity, impacts that have collapse durations of less than a microsecond the dynamic effect due to material inertia becomes unavoidable (as discussed in details in Part 2 [20]). In such cases, which is expectable for the collapse of very small bubbles, the estimated impact loads would be different from static FE approach. Moreover, if the target material is strain rate sensitive, the strain rate dependency should be taken into account for better estimation of impact loads, at least by extrapolating the material properties at higher strain rate corresponding to cavitation impact (as discussed in $[20,21])$.

Two types of elements CAX4R (continuum axisymmetric 4 nodes reduced integration element) and CINAX4 (continuum infinite axisymmetric 4 nodes element) were used for meshing. Zero radial displacement and symmetry axis rotation constraints have been used on the axis of symmetry. Infinite element at the bottom and right most side imposes condition of zero stress and zero strain at infinity. Fig. 1(a) shows meshing details. Bold black lines separate different sections. Fig. 1(b) shows magnified view of the loading region. The material behavior is modeled as elastic-plastic isotropic hardening and a Gaussian type of distribution models the impact pressure due to cavitation bubble collapse. FE computations are made using ABAQUS standard (version 6.11-2). 

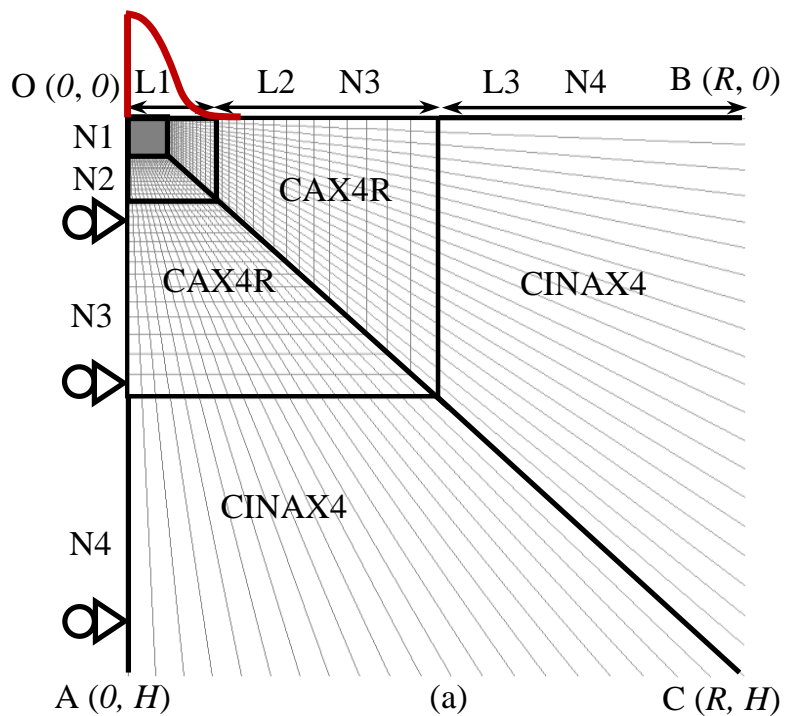

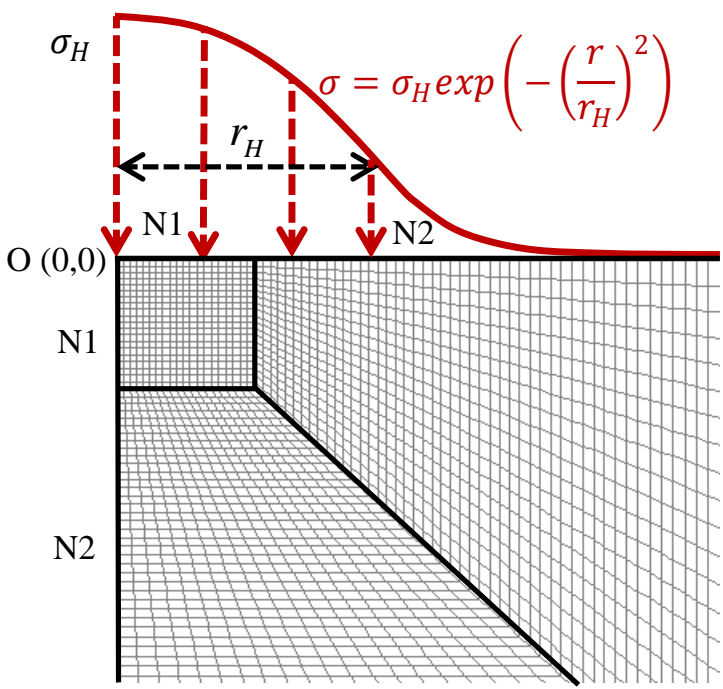

(b)

Fig. 1. (a) 2-D axisymmetric mesh used for numerical simulation. L represents length of one or more section as shown by arrow. N represents number of elements on the surface of different section. Number of elements (N1, N2, N3 and N4) in different section is always constant, but section lengths (L1, L2 and L3) are parameterized from radius of hydrodynamic pressure field, $r_{H}$, e.g. if $r_{H}=10 \mu \mathrm{m}, \mathrm{N1}=20, N 2=60, N 3=40$ and $N 4=1$ then, $L 1=1.5 \times r_{H}=15 \mu \mathrm{m}, L 1+L 2=L 3=R / 2=8 \times L 1=120 \mu \mathrm{m}, H=R=240 \mu \mathrm{m}$ using geometric progression with common ratio of 1.01. (b) Shows magnified view of the loading region. Minimum element size is $0.123 \mu \mathrm{m}$ for $r_{H}=10 \mu \mathrm{m}$.

The mesh has been verified against Hertz theory by applying Hertzian type of load [22] on to a purely elastic material (Young's modulus $=200 \mathrm{GPa}$ and Poisson's ratio $=0.3$ ) for which the analytical solution is known. As expected (Fig. 2), the maximum shear stress $\mathrm{T}_{\max }=0.31 \mathrm{P}_{0}$ occurs at a depth of $\mathrm{z}_{\max }=0.48 \mathrm{r}_{\mathrm{c}}$ along the axis of symmetry (OA) as defined in Fig. 1.

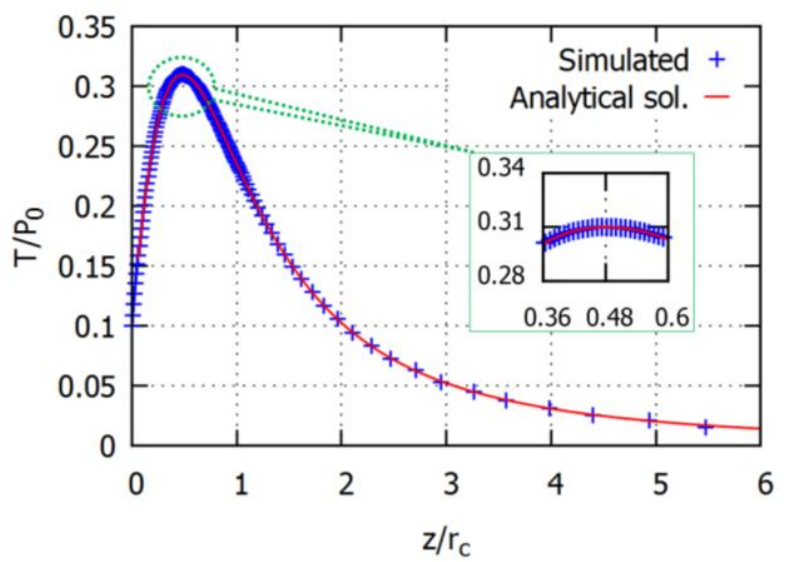

Fig. 2. Variation of maximum shear stress into the material along depth on the symmetry axis (OA). Maximum value of $T_{\max } / P_{0}$ is 0.31 at $\mathrm{z} / \mathrm{r}_{\mathrm{c}}=0.48$

\subsection{Hydrodynamic impact pressure distribution}

The impact pressure induced by the collapse of a cavitation bubble has a complex form in both space and time. It results from several unsteady mechanisms including the development of a microjet [23] and shock waves [24]. Ideally, the better option for the inverse FE technique would be to use the impact load generated from cavitation bubble collapse, and then optimize the parameters associated with bubble collapse in order to reproduce a given experimental pit numerically. To do so, fluid-structure interaction has to be considered and the authors in [5, 18] have highlighted the difficulties associated with such simulation of cavitation bubble collapse. In industrial applications as well as for the 
experiment considered here [15, 18], it is nearly impossible to use impact loads deduced from CFD (Computational Fluid Dynamics) since many parameters are unknown such as the location of bubble collapse, its initial size and the local collapse driving pressure gradient corresponding to each pit produced in cavitation pitting test. All of these parameters influence the impact load and can be finely controlled only in single bubble experiments.

The only feasible option would be to use a representative pressure field and analyze the material response to this load. A Gaussian type of distribution in space $\sigma(r)$ is considered for the hydrodynamic impact load generated by the collapse of a cavitation bubble, as given by Eq. 1 and shown in Fig. 3:

$$
\sigma=\sigma_{H} \exp \left(-\left(\frac{2 r}{d_{H}}\right)^{2}\right)
$$

Parameter $\sigma_{H}$ is the maximum amplitude of the hydrodynamic impact pressure, whereas radius $r_{H}=d_{H} / 2$ characterizes the radial extent of the load. Note that such a Gaussian law has already been used in $[5,19]$ for cavitation erosion studies. Moreover, in the current study it has been shown that normalized pit shapes numerically obtained by Gaussian types of pressure fields are very close to the experimental pit shapes, examined for a large number of pit, as will be discussed in Section 4.

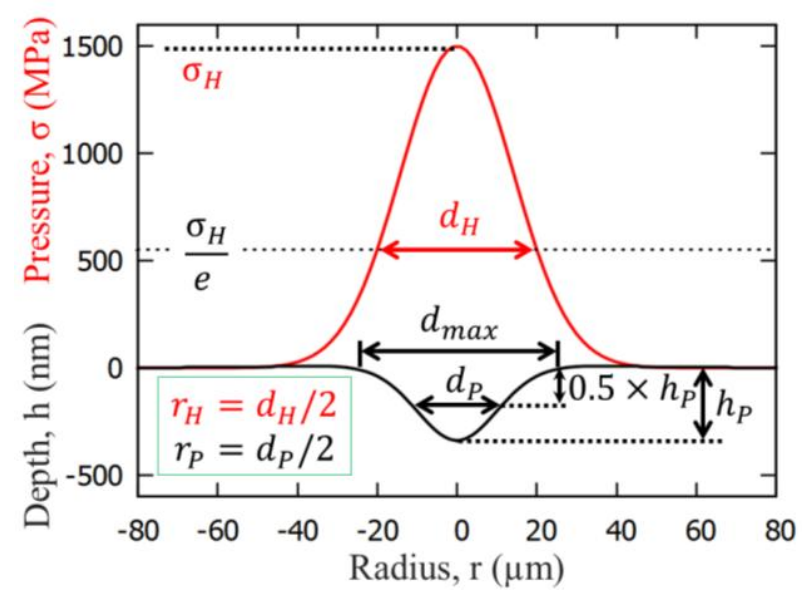

Fig. 3. Gaussian shape of the hydrodynamic impact pressure and resulted cavitation pit. $d_{\text {max }}$ represents the maximum diameter of the pit.

The residual plastic deformation after unloading representing a cavitation pit is characterized mainly by two parameters, the pit depth, $h_{P}$ and the pit diameter, $d_{P}$ as shown in Fig. 3. The pit diameter is measured at $50 \%$ of pit depth to be consistent with experimental data. The reason of this definition of the pit diameter is discussed in Section 4 . The pit depth, $h_{P}$, is the actual depth of the pit with respect to the original virgin surface. Since each pit is characterized here by only two parameters (depth and diameter), it seems reasonable to have only two free parameters to be optimized in the loading law.

\subsection{Constitutive equations of the material}

Numerical and experimental studies indicate that the strain rate involved in cavitation erosion process could be as high as $10^{5}$ or $10^{6} \mathrm{~s}^{-1}[3,4,20]$. So it is necessary to take into account the strain rate sensitivity of a material in the FE simulations. The current material Al-7075 has a very weak strain rate sensitivity as shown in [21], which could be neglected. Hence, the deformation behavior of the material Al-7075 was characterized by quasi-static uniaxial tensile tests performed at a strain rate of $4 \times 10^{-4} \mathrm{~s}^{-1}$. The corresponding true stress-true strain $(\sigma-\varepsilon)$ curve of the material is shown in Fig. 4. However, this curve corresponds to bulk tensile properties whereas we are interested in cavitation 
pitting which is a localized phenomenon. Depending on the microstructure, the bulk and local behavior of the material could be different, as addressed in $[20,21]$ and in that case the material properties should be replaced by the appropriate one, typically, obtained from nanoindentation tests.

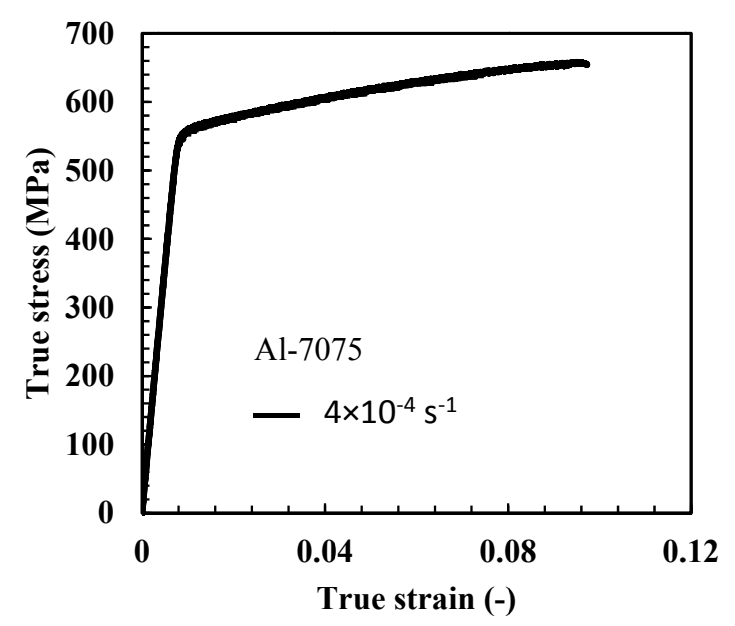

Fig. 4. True stress-true strain curve of Aluminum alloy 7075-T651 obtained by uniaxial tensile test.

The stress-strain curve can be represented by Ramberg-Osgood relationship [25] as given by Eq. (2) where the plastic part has been modified by Ludwik's equation [26] which includes yield stress $\left(\sigma_{y}\right)$.

$$
\varepsilon=\varepsilon_{e}+\varepsilon_{p}=\frac{\sigma}{E}+\left(\frac{\sigma-\sigma_{y}}{K_{y}}\right)^{m_{y}}
$$

$\varepsilon_{e}, \varepsilon_{p}$ and $E$ represent elastic strain, plastic strain and Young's modulus respectively. $K_{y}$ and $m_{y}$ are material constants obtained by least-square fitting of stress-strain curve in the plastic region. The criterion used for estimating the elastic limit corresponds to a ratio $\varepsilon_{p} / \varepsilon_{e}$ of the permanent plastic deformation $\varepsilon_{p}$ to the elastic deformation $\varepsilon_{e}$ equal to 5\% [25]. Material properties for the aluminum alloy considered in this paper are given in Table 1.

Table 1. Tensile properties of Aluminum alloy 7075-T651 (Al 7075)

\begin{tabular}{|ll|}
\hline Material & Al 7075 \\
Yield strength, $\sigma_{y}$ & $530 \mathrm{MPa}$ \\
Ultimate tensile strength, $\sigma_{u}$ & $645-660 \mathrm{MPa}$ \\
Modulus of elasticity, $E$ & $71.9 \mathrm{GPa}$ \\
Poisson ratio, $v$ & 0.33 \\
Ultimate strain, $\varepsilon_{u}$ & $0.086-0.095$ \\
Materials parameter, $K_{y}$ & $447 \mathrm{MPa}$ \\
Materials parameter, $m_{y}$ & 0.5 \\
\hline
\end{tabular}

To be consistent with the pitting test technique, no damage has been considered in the current study. As pitting tests were conducted within the incubation period to avoid any mass loss, pits are assumed to be formed by plastic deformation only.

\section{Simulation of cavitation pitting}

\subsection{Primary results}

A simulation campaign of 55 simulations has been carried out using the materials properties of Table 1 for different values of the size of the Gaussian pressure distribution, $d_{H}(10,20,40,60,80,100,120,140,160,200,240 \mu \mathrm{m})$ and of the peak Gaussian pressure, $\sigma_{H}(1.0,1.5,2.0,2.5$ and 3.0 GPa). The results are analyzed in terms of the deformed pit 
shape. Fig. 5 shows the applied pressure and the corresponding deformed geometry. As an example, Fig. 5(a) shows that pit depth and diameter both increase with size of impact loads. Whereas, with increase in peak pressure of hydrodynamic impacts of similar size as shown in Fig. 5(b), the pit depth increases significantly but the pit diameter does not change much. Same figure also shows that, at $\sigma_{H}=1 \mathrm{GPa}$, no pit forms i.e. the plastic deformation does not occur into the material although the yield strength of the material is $530 \mathrm{MPa}$. This is because the von Mises equivalent stress induced into the material does not exceed the yield strength, which is a direct consequence of the plasticity confinement, similar to indentation induced plasticity. At $\sigma_{H}=3 \mathrm{GPa}$ when $\mathrm{d}_{\mathrm{H}}>40 \mu \mathrm{m}$, the pit depth becomes significantly higher than the measured experimental maximum values of about $10 \mu \mathrm{m}$ (for example see Fig. 15). The observations from Fig. 5 indicate that deep pits of small diameters are most probably formed by impacts of small diameter but high peak pressure.

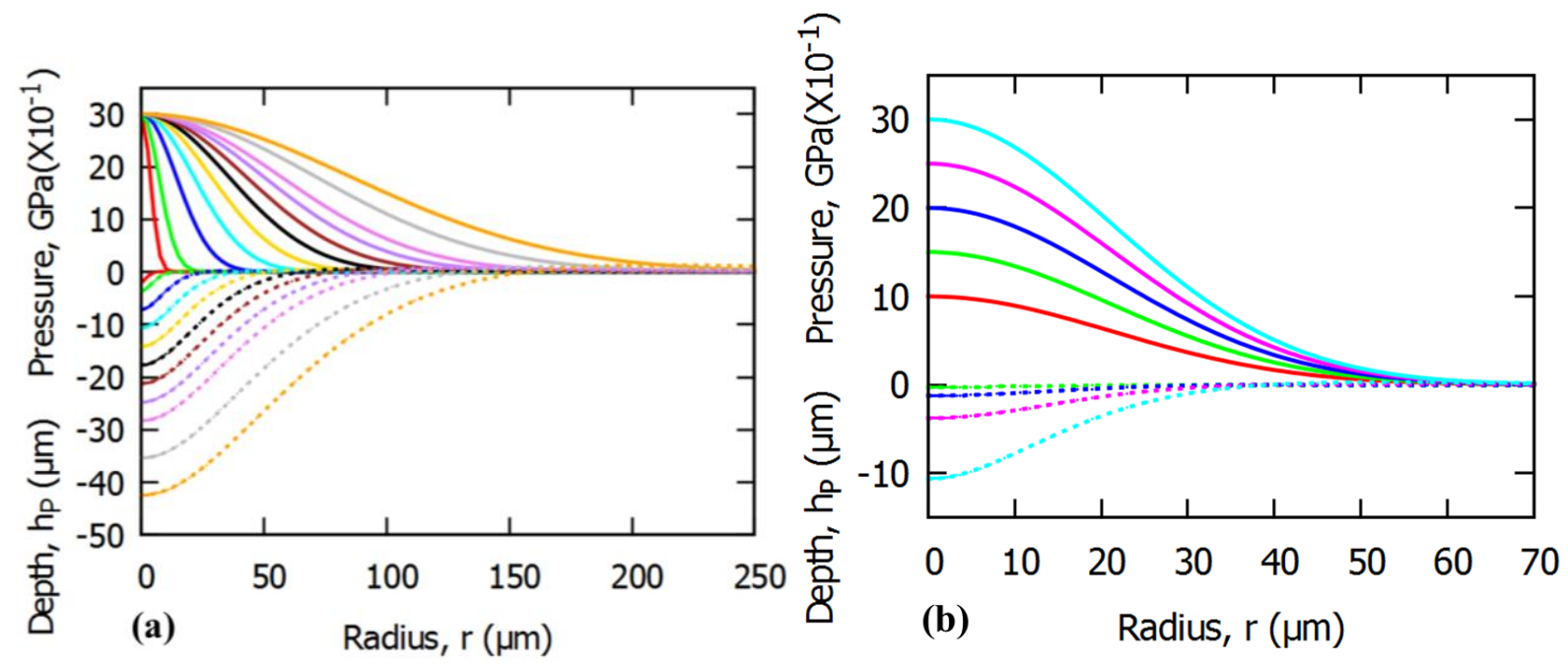

Fig. 5. Applied Gaussian loads and corresponding unloaded deformed geometry represent simulated pit shape. (a) At $\sigma_{H}=3 \mathrm{GPa}$ for $r_{H}=5$ to $120 \mu \mathrm{m}$ and (b) At $r_{H}=30 \mu \mathrm{m}$ for $\sigma_{H}=1$ to $3 \mathrm{GPa}$, no pit formed at 1 $G P a$.

In Fig. 6 all simulated pit depths and diameters are plotted where dotted line connects pit data for iso- $r_{H}$ and continuous line connects pit data for iso- $\sigma_{H}$. As can be seen along the iso- $\sigma_{H}$, the pit depth, $h_{P}$ shows a linear behavior with the pit diameter, $d_{P}$. For a given $\sigma_{H}$, the pit depth increases with $\mathrm{d}_{\mathrm{H}}$ and conversely, for a given $\mathrm{d}_{\mathrm{H}}$ the pit depth increases with $\sigma_{H}$. Thus, deep pits of large diameter may be caused by impacts of bigger size at relatively low pressure.

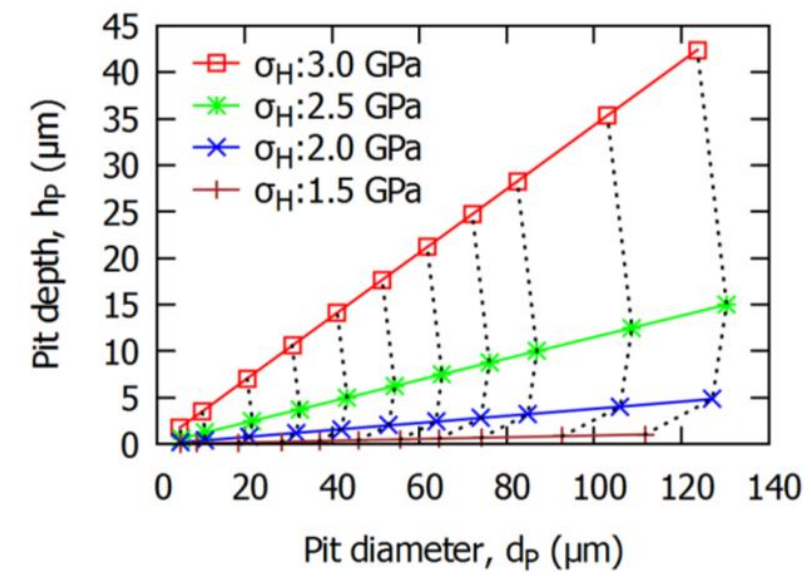

Fig. 6. Simulated pit depths and diameters show the effect of $\sigma_{H}$ (solid lines) and $r_{H}$ (dotted lines) 
One interesting fact in Fig. 6 is that the solution appears to be bijective since the lines passing through different iso$\sigma_{H}$ or iso- $r_{H}$ data points do not intersect themselves. Thus, it is evident that a pit of a particular depth, $h_{P}$ and diameter, $d_{P}$ is formed by a unique impact of particular size, $\mathrm{d}_{\mathrm{H}}$ and peak pressure, $\sigma_{H}$. Obviously, the data lines for different $\sigma_{H}$ converge towards zero when $r_{H}$ goes to zero. Interestingly, such bijective behavior is also observed in the case of dynamic explicit analysis of cavitation pitting as presented in Part 2 paper [20].

A more convenient representation of the curves in Fig. 6, is given in Fig. 7 where the ratio $h_{P} / d_{P}$ (pit shape factor) is plotted versus the pit diameter, $d_{P}$. One should notice that the iso- $\sigma_{H}$ curves are now horizontal and the iso- $r_{H}$ curves are quasi-vertical except for the highest values of $r_{H}$.

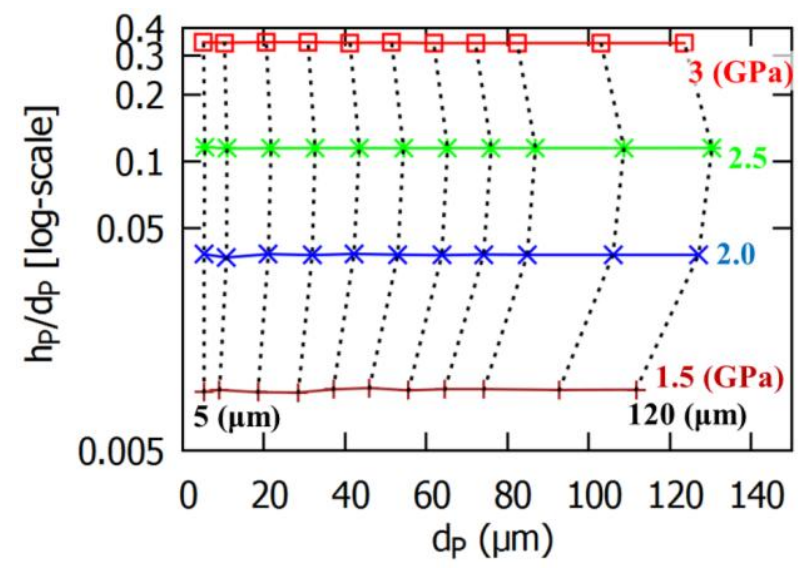

Fig. 7. Variation of shape factor and diameter of simulated pits with $\sigma_{H}$ (continuous lines) and $r_{H}$ (dashed lines).

From the simulation campaign (as given by Eq. (3)), we also observed that the ratio of pit diameter, $d_{P}$ to diameter of hydrodynamic impact, $d_{H}$ remains almost constant irrespective of values of $\sigma_{H}$ and $r_{H}$.

$$
\frac{d_{P}}{d_{H}}=\frac{r_{P}}{r_{H}} \approx k
$$

Here $k$ is a constant that depends on material only. For Al 7075, $k$ is approximately 0.52 .

Finally, we could establish from Fig. 7 that the pit shape factor, $h_{P} / d_{P}$ follows a power law behavior which can be written by the following equation:

$$
\frac{h_{P}}{d_{P}} \approx\left(\frac{\sigma_{H}}{\sigma^{*}}\right)^{\beta}
$$

Here $\sigma^{*}$ and $\beta$ are two constants that depend on the material only. The material parameter $\sigma^{*}$ has same unit as $\sigma_{H}$ and $\beta$ is dimensionless. For $\mathrm{Al} 7075, \sigma^{*}$ and $\beta$ are found to be $3600 \mathrm{MPa}$ and 5.8 respectively.

Combining Eq. (3) and (4), we can write Eq. (5) as follows-

$$
\ln \left(\frac{d_{H}}{h_{P}}\right)=\beta \ln \left(\frac{\sigma^{*}}{\sigma_{H}}\right)+\ln \left(\frac{1}{k}\right)
$$

Eq. (5) represents a linear equation on log-log graphs. Fig. 8 shows the plot of $\sigma^{*} / \sigma_{H}$ versus $d_{H} / h_{P}$ for all the simulated data $\left(\sigma_{H}=1.5\right.$ to $3 \mathrm{GPa}$ and $r_{H}=5$ to $\left.120 \mu \mathrm{m}\right)$ and interestingly they all follow a unique linear behavior on log-log graphs. Thus, Fig. 8 validates Eq. (5) or in other words Eq. (3) and (4). 


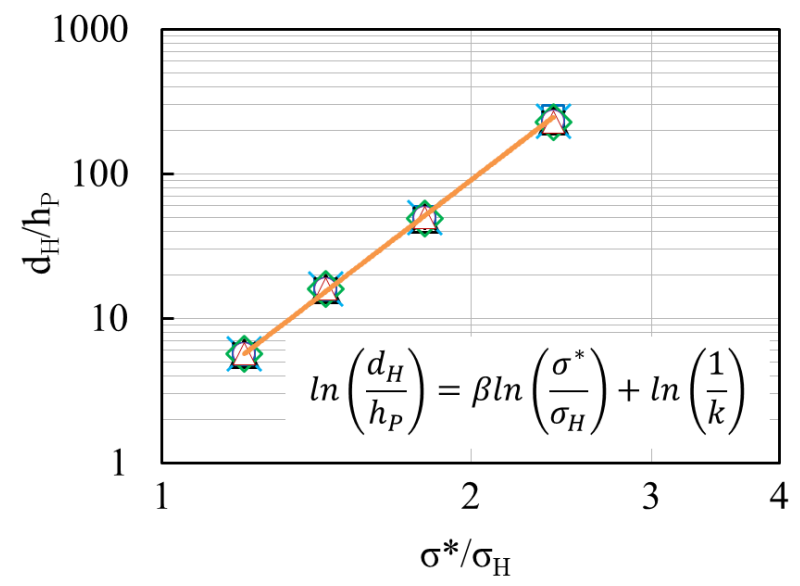

Fig. 8. Plot of $\sigma^{*} / \sigma_{H}$ versus $d_{H} / h_{P}$ for all simulated data for $\sigma_{H}=1.5$ to $3 \mathrm{GPa}$ and $r_{H}=5$ to $120 \mu \mathrm{m}$. Linear behavior on log-log scale.

Now by using Eq. (3) and (4), $\sigma_{H}$ and $d_{H}$ can be predicted when pit depth and diameter are known for experimental pits. Prediction of $\sigma_{H}$ and $d_{H}$ for a particular given pit using Eq. (3) and (4) will be termed as analytical method throughout the paper.

\subsection{Verification of the model predictions}

In order to verify Eqs. (3) and (4), six experimental pits of given depths and diameters have been chosen arbitrarily. Simulations were conducted with input parameters of the applied pressure field $\sigma_{H}$ and $d_{H}$ estimated using Eq. (3) and (4) with the material constants obtained in Section 3.1. Fig. 9 shows the comparison of the simulated pit depths and diameters with the original values. It indicates a very good ability of the model although some deviations are evidenced for the biggest pits. This comes from the constant factor, k, used in Eq. (7). We could observe from the simulations that the pit diameter is actually a more complex function of both pressure size and peak pressure of hydrodynamic impact. This motivates the development of an alternative and more accurate numerical inverse method to directly estimate peak pressure and pressure size for a given pit depth and diameter as discussed in Section 5, using the model predictions for the initial guess of the optimization algorithm.

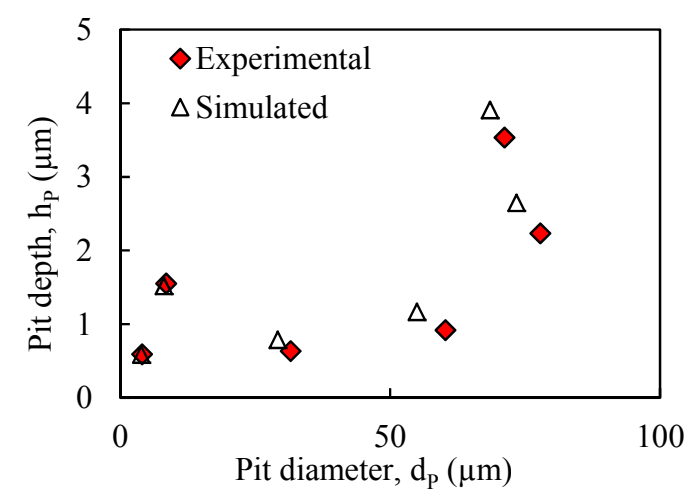

Fig. 9. Comparison of experimental pit depth and diameter with that of simulated. Input parameters for simulation $\sigma_{H}$ and $r_{H}$ were predicted using analytical method (Eq. (3) and (4)). 


\section{$4 \quad$ Pitting test analysis}

\subsection{Pit analysis method}

Pitting tests were conducted on aluminum alloy 7075-T651 in a cavitation tunnel at flow pressure of 10, 15, 20, 30 and 40 bar within the incubation period (test method is described in details in [27]). The surface of the samples prior to test were prepared following conventional metallurgical polishing method down to a mirror polishing with diamond paste of $0.25 \mu \mathrm{m}$. The surfaces of the pitting tested samples have been analyzed using a conventional contact profilometer (microprobe radius $2 \mu \mathrm{m}$ ). Different regions of $2 \mathrm{~mm} \times 4 \mathrm{~mm}$ with a mesh size of $1 \mu \mathrm{m} \times 1 \mu \mathrm{m}$ had been scanned on the sample surface where maximum pitting occurs. Different regions were scanned to have a sufficient number of pit data for the statistical analysis. The scanned numerical surfaces were analyzed using a Matlab script to estimate pit depth $\left(h_{P}\right)$ and diameter $\left(d_{P}\right)$. The micro-probe of radius $2 \mu \mathrm{m}$ used here might cause some errors in the estimation of pit parameters, especially for smaller pit with bigger depth. In contrary, as can be seen in Fig. 15 the fact that most of the pits are far bigger in diameter than depth indicates that such error could be negligible, at least from statistical point of view for large number of data. In [7], Franc et al. have done similar analysis of pits using constant cut-off depth (0.5 $\mu \mathrm{m})$ and highlighted the associated difficulties arising from the complex nature of pitting. The use of a cut-off depth was necessary to avoid surface noise or roughness, while estimating the pit diameter. In the current analysis also, the same raw data as obtained by Franc et al. [7] using the same contact profilometer have been utilized.

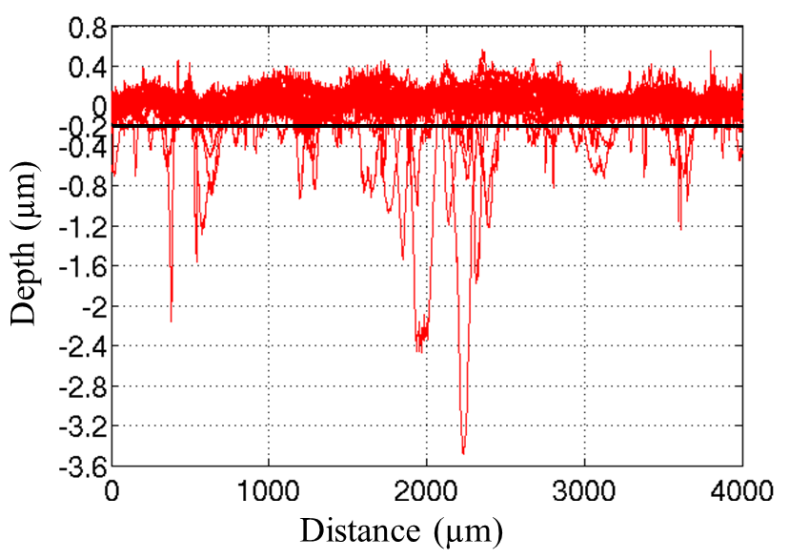

Fig. 10. Twenty profiles along different lines on the pitting tested surface are plotted together. Maximum noise level is approximately -0.2 $\mathrm{um}$ as shown by black the line. Al-7075 material cavitation tested at flow pressure of 40 bar for $2 \mathrm{sec}$.

Firstly, the pits are detected from the part of the profiles below a cut-off depth of $0.5 \mu \mathrm{m}$ in order to avoid the surface noise. For all Al 7075 samples, the maximum level of noise was found to be approximately $0.2 \mu \mathrm{m}$, as shown in Fig. 10 . Once the pits are all detected, the pit depth is always taken as the actual depth with respect to the virgin surface. The pit diameter is then measured at a depth chosen as a fraction of the pit depth (typically $50 \%$ ). The use of such a fractional cut-off depth has many advantages over a fixed cut-off depth: (1) the measured pit diameter does not depend on the arbitrary cut-off depth and thereby avoids any size effect, (2) it is a more appropriate way of measuring the pit diameter as it exclude the ambiguity involved in the choice of any arbitrary the cut-off depth and (3) statistical analysis of measured pit depths and diameters provide more accurate results. Finally, the use of a fractional cut-off depth is also favorable for the numerical inverse method presented in Section 5. 


\subsection{Typical results}

Systematic analyses of pits were done in order to understand the influences of two different methods namely fractional cut-off depth (FCOD) method and constant cut-off depth (CCOD) method. As an example, Fig. 11 shows the influence of the analysis method on pit boundaries. Fig. 11(a) represents the pit boundaries obtained by CCOD method using a cut-off depth of $0.5 \mu \mathrm{m}$. Fig. 11(b)-(d) represent the same pit boundaries at 20\%, 40\% and 50\% fraction cut-off depth respectively. It is clear from the figures that with increase in FCOD, the problem of overestimation of the pit diameter due to overlapping of pits is reduced. After analyzing all the surfaces of different cavitation tests, it is found that at 50 $\%$ of FCOD there is almost no overlapping of pits, which have been detected by using CCOD of $0.5 \mu \mathrm{m}$. Selection of FCOD is related to the CCOD used for pit detection. The condition to avoid over estimation of pit diameter (especially for shallow pit) is that the $(\mathrm{FCOD} \times \mathrm{CCOD})$ or $(\mathrm{FCOD} \times$ pit depth) should be greater than noise level of the tested surface. This condition is fulfilled here since FCOD $=50 \%$ and $\mathrm{CCOD}=0.5 \mu \mathrm{m}$ so that FCOD $\times \mathrm{CCOD}=0.25 \mu \mathrm{m}$ whereas noise level was determined as $0.2 \mu \mathrm{m}$. This condition is not fulfilled for Fig. 11(b) where FCOD $\times$ CCOD $=$ $0.1 \mu \mathrm{m}$, which explains the bad definition of pits.
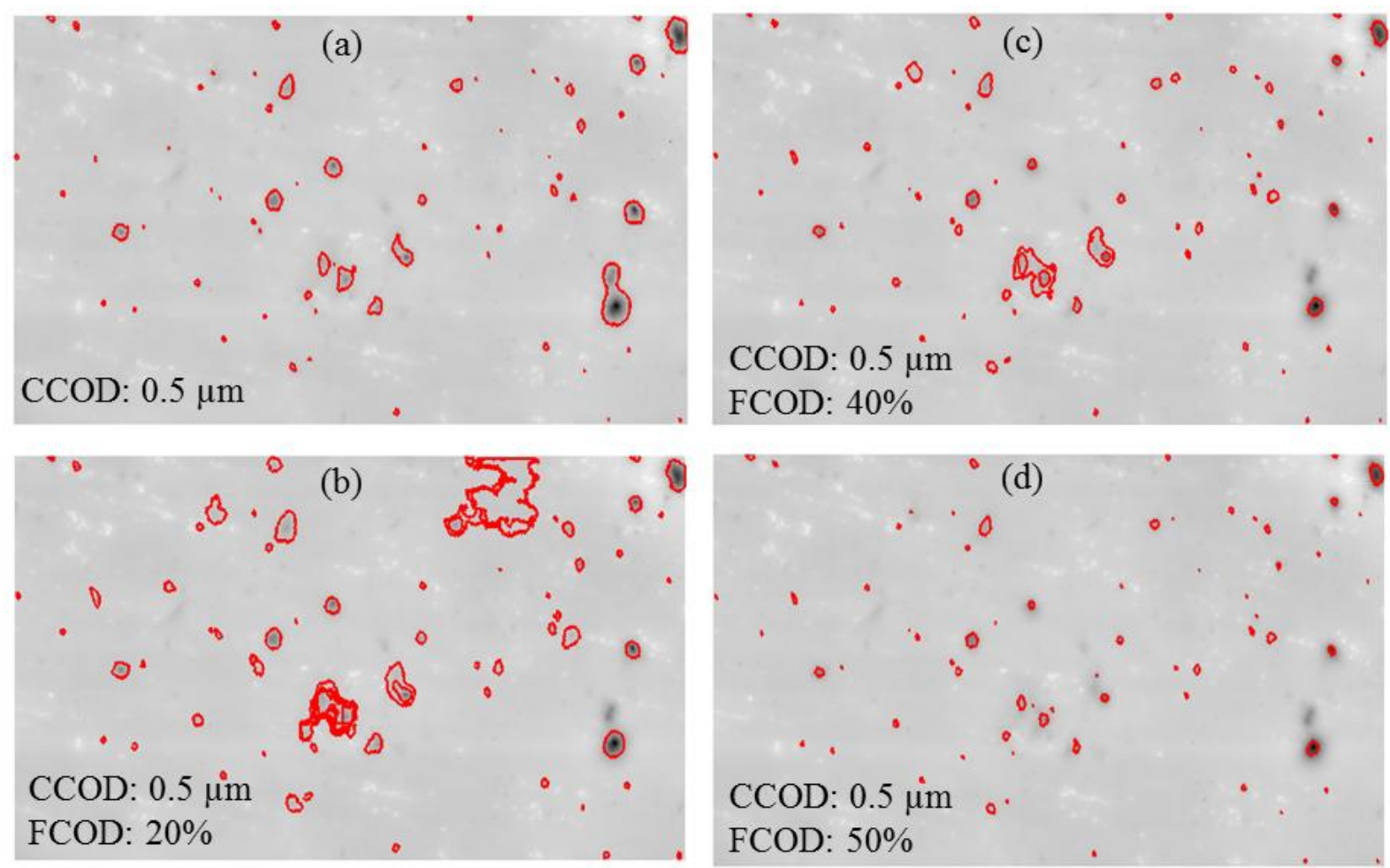

Fig. 11. Comparison of fractional and constant cut-off depth method for pit parameter determination, (a) CCOD method and (b)-(d) FCOD method at 20\%, 40\% and 50\% cut-off depth respectively. Gary scale is an indicator of pit depth. Cavitation test: Al 7075 at 40 bar for 2 sec.

As direct measurement of maximum pit diameter $\left(d_{\text {max }}\right)$ at zero-level by using any of the two methods is not possible due to noise and overlapping, focuses were made to an alternative method as discussed in next Section 4.3.

\subsection{Analysis of non-overlapping pits}

In order to identify the relevance of the Gaussian pressure field assumed, simulated and experimental pit shapes have been compared. Twenty pits almost unaffected by surface noise or overlapping have been identified, one example is shown in Fig. 12. As can be seen, the pit profile is not closed at zero-level, this is because of the way the pit is being 
detected to avoid surface noise detection. Hence, the maximum pit diameter at zero-level was estimated by linear extrapolation of pit diameters at 5\% and 10\% of pit depth. In Fig. 13 normalized pit shape i.e. pit depth normalized by maximum pit depth vs. pit diameter normalized by maximum pit diameter for the 20 experimental and all (55) simulated pits have been plotted together. The normalized pit shapes are almost the same for both simulated and experimental pits. The little variation in experimental data could be due to error associated with the measurement of maximum pit diameter, as well as shape correlation between the pit profile and the profilometer microprobe used for the measurement. This particular observation depicts that the Gaussian pressure field describes reasonably well the effective pressure field associated with bubbles collapse.

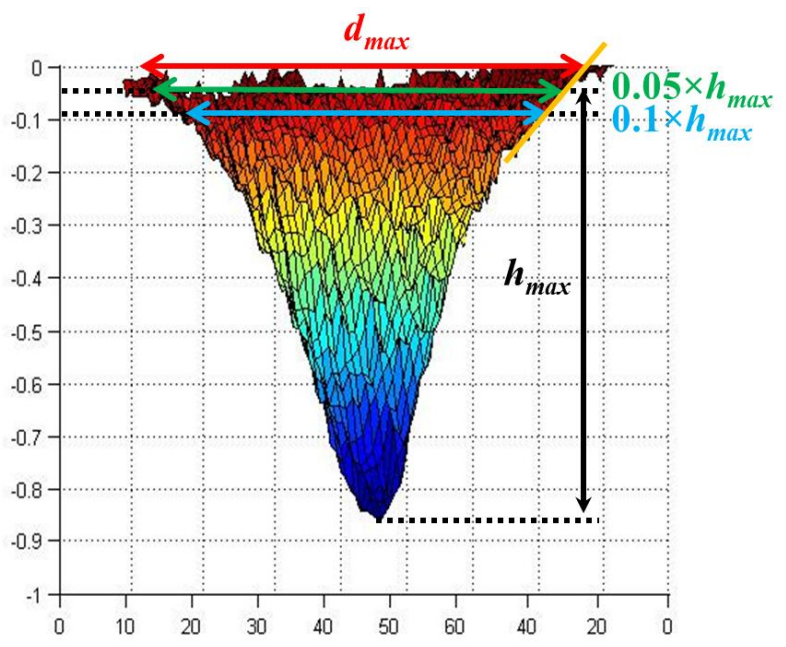

Fig. 12. An experimental pit obtained by contact surface profilometer with microprobe of radius $2 \mu m$. $d$ : diameter, $h$ : depth, max: maximum.

Now as shown in Fig. 13 by black unfilled points, a common normalized pit shape (mean of the simulated curves) could be considered to estimate $d_{\max }$ and for a particular material we can write

$$
\frac{d}{d_{\max }}=f\left(\frac{h}{h_{\max }}\right)
$$

Using Eq. (6) $d_{\max }$ for the experimental pit can easily be predicted, as $d$ is already estimated by using FCOD method. For FCOD of $50 \%$ i.e. $h / h_{\max }=0.5$, the corresponding value of $d / d_{\max }$ is $\sim 0.416$ as can be seen in Fig. 13 . 


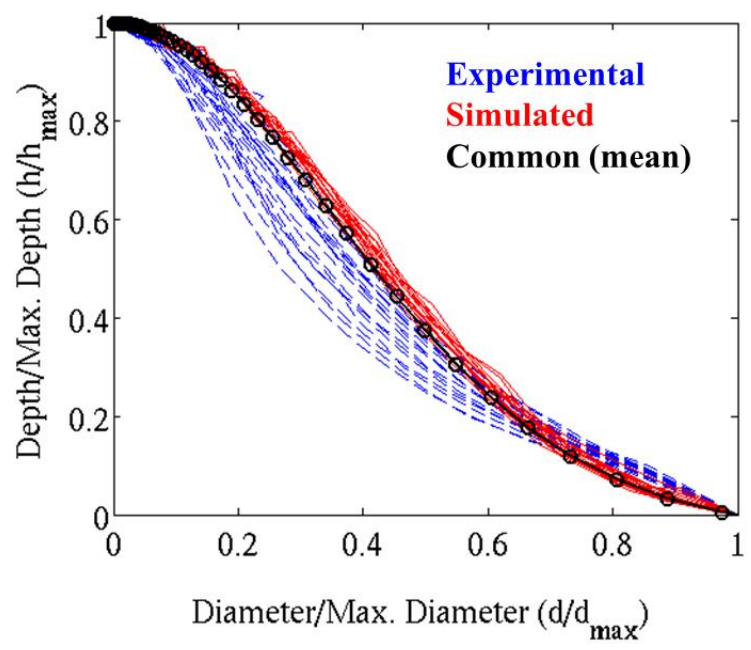

Fig. 13. Normalized pit shape of simulated and experimental pits. Twenty non-overlapping experimental pits were identified almost unaffected by surface roughness.

\section{Numerical inverse method}

\subsection{Principle}

The principle of the inverse FE technique, as termed here Numerical Inverse Method (NIM), to estimate impact load parameters- peak pressure $\left(\sigma_{H}\right)$ and its radial extent $\left(r_{H}\right)$ from pit parameters- depth $\left(h_{P}\right)$ and diameter $\left(d_{P}\right)$ is explained here. The method is based on simple interpolation technique, but found to be very efficient to implement on a large number of data required for statistical analysis. The one-to-one correspondence between set of $\left(\sigma_{H}, r_{H}\right)$ and $\left(h_{P}, d_{P}\right)$ as mentioned in Section 3.1 makes it possible to implement the method. The basic principle of the method is to run ABAQUS simulation from an initial guess of $\sigma_{H}$ and $r_{H}$, compare the computed pit depth and diameter with experimental values and iterate until they are optimized for a given error limit in pit dimensions. Similar to the experimental pit diameter, the simulated pit diameter was also measured at 50\% of pit depth for consistency in defining the pit diameter. Let us mention here that, if we use a constant cut-off depth of $0.5 \mu \mathrm{m}$ (instead of FCOD of $50 \%$ ) to estimate the simulated pit diameter, then during optimization if the simulated pit depth becomes smaller than $0.5 \mu \mathrm{m}$, there will be no value for the pit diameter and the optimization method will not work for that pit. This is one more reason for considering a fractional cut-off depth rather than a constant one.

The initial guess is an important factor that influences the number of simulations required to obtain the solution. The optimization method is discussed graphically in details in this section.

In Fig. 7 each data point represents a FE simulation results for which all four parameters $\left[d_{P}, h_{P} / d_{P}, \sigma_{H}\right.$ and $\left.d_{H}\right]$ are known. Continuous solid line connects data points for iso- $\sigma_{H}$ and dotted line connects iso- $d_{H}$ data points. More details related to the figure can be found in Section 3.1. These known simulated data have been used for the optimization method.

The optimization method starts with finding out of a triangle (such as $\mathbf{\Delta} 123$ on the base in Fig. 14) from the simulated data points (from Fig. 7) which ensures that the experimental data point (for which only $d_{P}$ and $h_{P} / d_{P}$ is known) always lies inside the triangle. Now to predict $\sigma_{H}$ for the experimental data point, a three dimensional (3D) plane (Fig. $14)$ is constructed from the three data points of the triangle. The $3 \mathrm{D}$ space is characterized by $d_{P}$ as $\mathrm{x}-\mathrm{axis}, h_{P} / d_{P}$ as $\mathrm{y}-$ axis and $\sigma_{H}$ as z-axis. For the prediction of $\sigma_{H}$ for the experimental data point, it is assumed that the point would lie on the $3 \mathrm{D}$ plane, hence can be estimated easily by interpolation. 


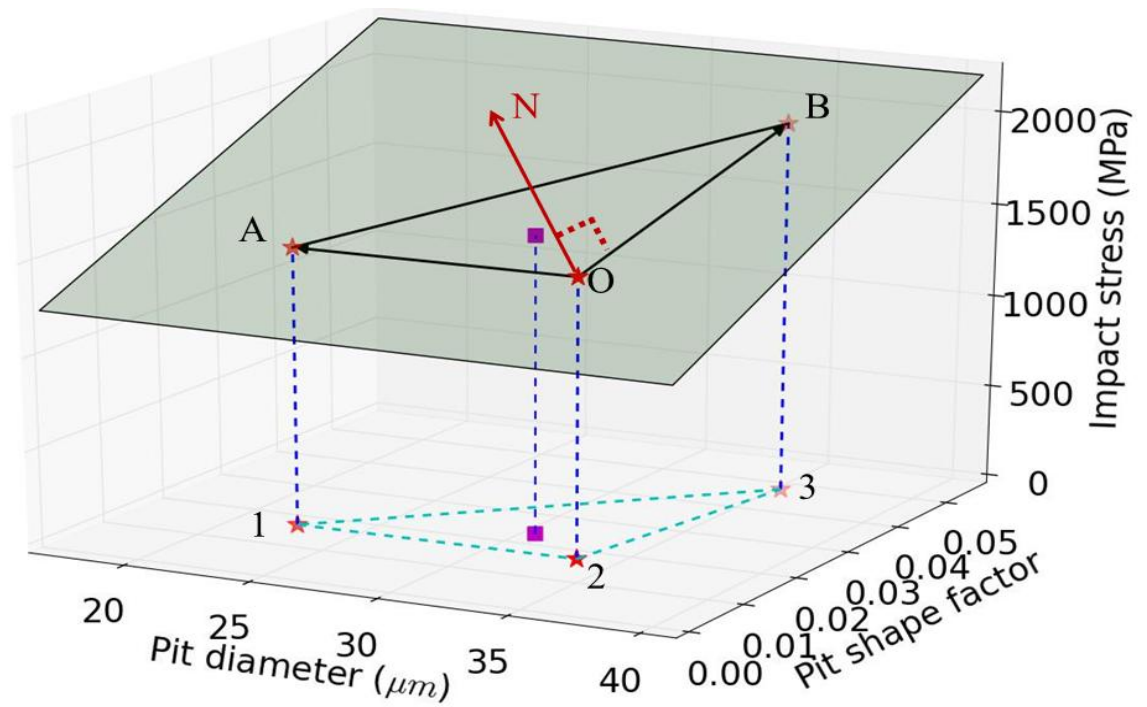

Fig. 14. Method of construction of $3 D$ plane through three points from the known results of simulated data points for the prediction of $\sigma_{H}$. Similar plane is also constructed to predict $d_{H}$ by considering the $z$-axis as $\sigma_{H}$.

Similarly, for the prediction of $d_{H}$ the $3 \mathrm{D}$ space is characterized by $d_{P}$ as x-axis, $h_{P} / d_{P}$ as y-axis and $d_{H}$ as z-axis.

Once $\sigma_{H}$ and $d_{H}$ are predicted, one FE simulation will be done and two errors are evaluated: error in pit depth and in pit diameter. If both errors are below a given value (typically $1 \mu \mathrm{m}$ in diameter and $0.05 \mu \mathrm{m}$ in depth) then the solution is assumed to be achieved, otherwise the process will continue by the selection of a new triangle for the prediction of $\sigma_{H}$ and $d_{H}$ until an acceptable solution is obtained. It should be noted that each time an FE simulation is done a new data point is added up into the database i.e. Fig. 7, for which the solution is known. Thus, the new triangle would become smaller and closer to the experimental data point, thereby the error domain is reduced improving the prediction accuracy.

\subsection{Convergence and accuracy}

It was observed that on average three simulations were needed for a given error limit of $1.5 \mu \mathrm{m}$ in pit diameter and 0.05 $\mu \mathrm{m}$ in pit depth. Time required for an ABAQUS simulation to be completed varies from 1-3 minutes depending upon the size of the problem. Thus to obtain optimized $\sigma_{H}$ and $d_{H}$ for a given experimental pit, average time required varies from 3-9 minutes. This is fairly economic since to optimize 200 pits, the total time required varies from 10-30 hours on a computer system with $24 \mathrm{~GB}$ ram and $2.8 \mathrm{GHz}$ Intel X5660 processor. Two hundred pits is a typical number of pits necessary for a reliable statistical analysis of a pitting test. 


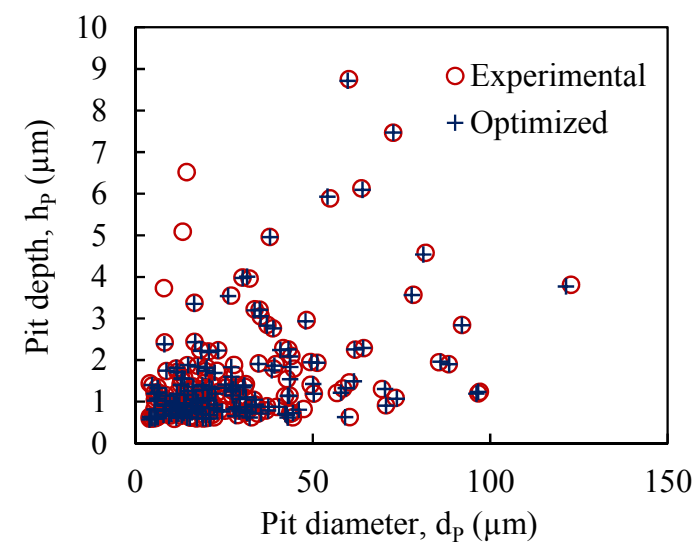

Fig. 15. Comparison of pit depth and diameter for experimental and optimized pit, cavitation test condition: upstream pressure 40 bar and exposure time $2 \mathrm{sec}$.

As an example, Fig. 15 shows comparison of experimental and simulated pit depth and diameter. Simulated data represent pit depth and diameter when solution is optimized. Out of 178 pits, all pits were solved very accurately other than three pits, which are difficult to solve. The difficulty arises when a pit has very high shape factor. In such cases, strain into the material goes beyond the maximum strain defined in material property for simulation and crushes the simulation. Occurrence of this problem is rare provided the exposure time in cavitation pitting test was sufficiently less to avoid overlapping of pits.

\section{Discussion}

The hydrodynamic peak pressure $\left(\sigma_{H}\right)$ and size $\left(d_{H}\right)$ corresponding to each pit at different flow pressures have been estimated using the numerical inverse method, as discussed in Section 5. As an example, Fig. 16 shows the values of $\sigma_{H}$ and $d_{H}$ corresponding to all pits at the flow pressure of 40 bar. Such a distribution of impact loads as a function of stress and size is considered here as an estimate of the cavitation intensity also called aggressiveness of the cavitating flow. Even though it was determined using a given material on which a pitting test was carried out, it is expected to characterize the only liquid flow. This was checked in [21] by considering different materials.

Such a distribution of impact loads gives representative loading conditions for this particular flow that can later be used in a FE simulation in order to predict the long-term behavior of the material. The principle of such a simulation would be to apply repetitively, on the material surface, the whole spectrum of impact loads a large number of times by randomly choosing the impact point for each load. By introducing an appropriate damage model of the material, it should be possible to predict the evolution of mass loss versus the exposure time [28]. 


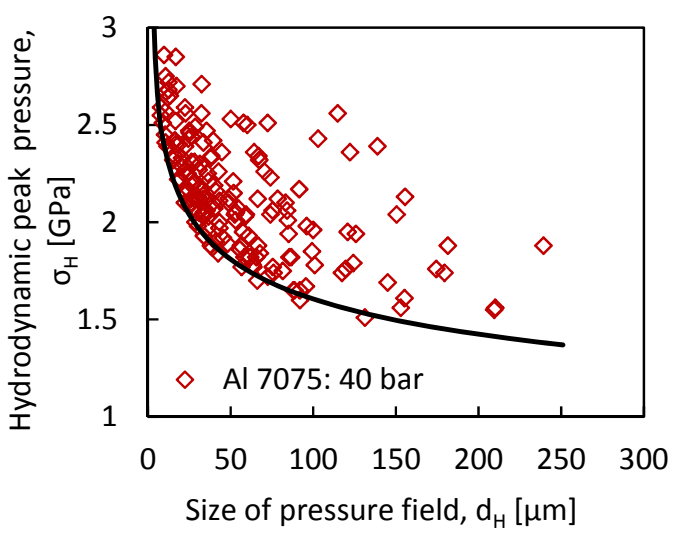

Fig. 16. Hydrodynamic peak pressure and sizes for all pits formed in Al 7075 under cavitating flow at 40 bar for $2 \mathrm{sec}$. Minimum depth of pit detected is greater than $0.5 \mu \mathrm{m}$.

As can be seen in Fig. 16, impacts of smaller size are associated with higher peak pressure and in most of the cases, as the impact size increases the peak pressure decreases. For a given size of impact, there exists a limit to the minimum value of associated pressure to form a cavitation pit deeper than the detectable depth. In the current study, $0.5 \mu \mathrm{m}$ cutoff depth $\left(h_{c}\right)$ is used for detection of pits or, in other words, minimum measured pit depth is close to $0.5 \mu \mathrm{m}$. The variation of limiting minimum value of peak pressure $\left(\sigma_{H_{\min }}\right)$ with impact size $\left(d_{H}\right)$ for a given cut-off depth $\left(\mathrm{h}_{\mathrm{c}}\right)$ follows a power law behavior and can be derived from Eq. (3) and (4) as shown in Eq. (7).

$$
\sigma_{H_{\min }}=\sigma^{*}\left(\frac{h_{c}}{k d_{H}}\right)^{1 / \beta}
$$

Eq. (7) is plotted in Fig. 16 (solid line) for $h_{c}=0.5 \mu \mathrm{m}$ with the same values of material constants $\sigma^{*}, k$ and $\beta$ as obtained earlier in Section 3.1 and is found to clearly define the lower bound of the data points. Eq. (7) does not change with flow pressure or velocity of cavitating fluid as all the parameters depend on material only. This can be verified from Fig. 17 as the distribution of $\sigma_{H}$ and $d_{H}$ for different cavitating flows, 10 bar and 40 bar, shows identical lower bound. In addition, Fig. 17 does not exhibit a clear influence of the operating pressure on the values of the impact pressures and sizes since the material was the same for the two flow conditions.

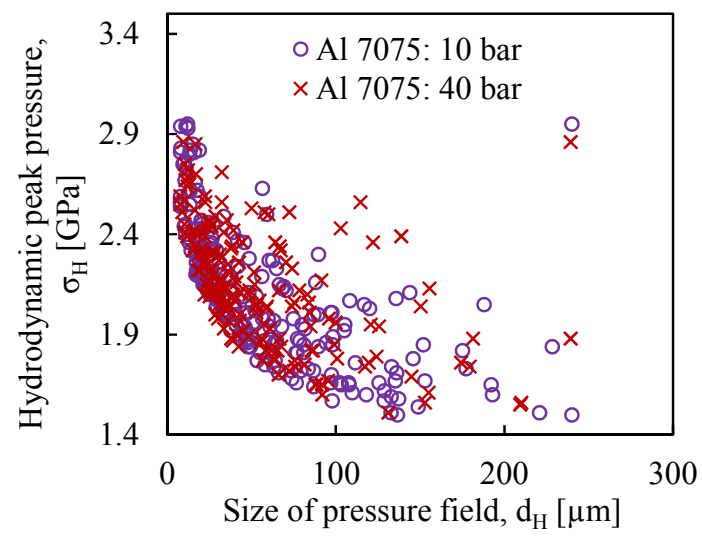

Fig. 17. Distribution of peak hydrodynamic pressures and corresponding sizes of pressure field associated with bubbles formed at different flow velocity 10 bar and 40 bar.

With increase in flow velocity, cumulative frequency of hydrodynamic impacts increases predominantly as shown in Fig. 18 and Fig. 19. Fig. 18 represents the cumulative frequency of impacts per unit area and unit time having peak 
pressure $\sigma_{H}$ greater than any given value on the horizontal axis, whereas Fig. 19 represents cumulative frequency of impacts per unit area and unit time having size of the pressure field greater than any given value on the horizontal axis. On a semi-log plot, the observed linear behavior indicates an exponential expression for the cumulative frequency of impacts versus the impact diameter $d_{H}$.

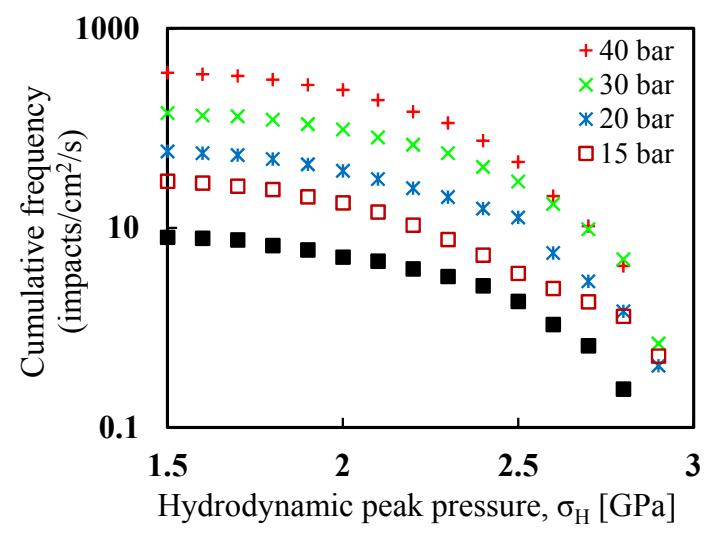

Fig. 18. Influence of cavitation flow pressure on cumulative frequency of impact per unit area and unit time having peak pressure $\sigma_{H}$ greater than any given value. The vertical axis is on log scale.

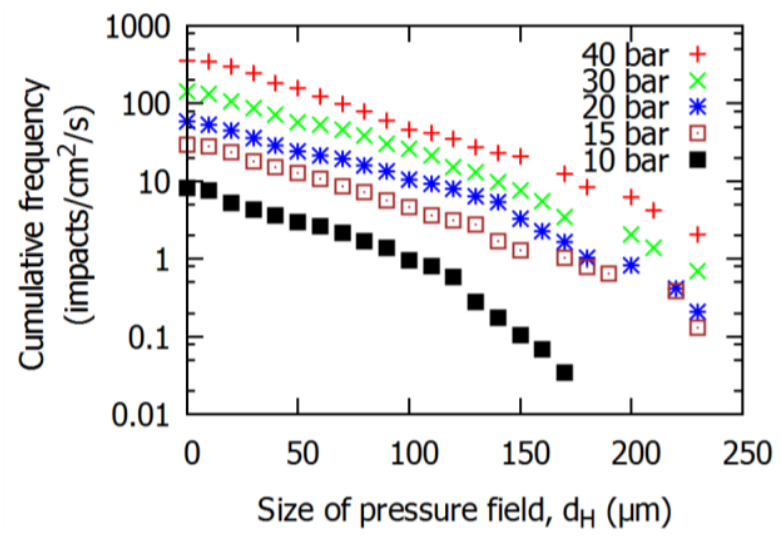

Fig. 19. Influence of cavitation flow pressure on cumulative frequency of bubbles per unit area and unit time having size of the pressure field greater than any given value. Almost linear plot on semi-log scale represent exponential behavior.

Simulation of cavitation pitting (Section 3.1) shows that no pit forms at 1 GPa peak pressure and from Fig. 18 it is also clear that peak stress did not reach $3 \mathrm{GPa}$ for any of the experimental pits obtained under different flow velocities. For all the pits detected by $0.5 \mu$ m cut-off depth, the peak pressure varies from $1.5 \mathrm{GPa}$ to less than $3 \mathrm{GPa}$. The probability of occurrence of pressure peaks of higher amplitude decreases rapidly. From a statistical point of view, the estimated values of impact loads are in good agreement with the previously obtained impact loads by Carnelli et al [13], although the material properties were characterized differently and the method of load estimation was different.

\section{$7 \quad$ Summary and conclusion}

One of the primary aims of the current paper was a better estimation of depth and diameter of cavitation pits formed under different cavitating flows. A cut-off depth is required to avoid error in the measurements of the pits due to the surface noise. The use of a fractional cut-off depth (FCOD) method to estimate the pit diameter is found to be better than the conventional constant cut-off depth (CCOD) method where the pit diameter depends on the choice of the cutoff depth 
The second objective of the current study was to estimate the hydrodynamic peak pressure $\left(\sigma_{H}\right)$ and the size of pressure field $\left(d_{H}\right)$ associated with a single bubble collapse that led to a pit of a given depth and diameter. Two methods namely analytical inverse method and numerical inverse method have been developed. The first analytical method predicts $\sigma_{H}$ and $d_{H}$ from pit depth and diameter using simple equations - (Eq. (3) and Eq. (4)). The second method is based upon a numerical technique that provides $\sigma_{H}$ and $d_{H}$ after numerically reproducing a given experimental pit and is found to be more accurate than the analytical one.

The impact load parameters $\left(\sigma_{H}, d_{H}\right)$ corresponding to all pits obtained from a pitting test can be considered as the signature of the cavitating flow and a measure of its aggressiveness. Such a combination of an experimental and a numerical method based on both pitting tests and FE computations provides an alternative to direct measurement of impact loads using conventional pressure sensors that present serious limitations as mentioned in the introduction.

Apart from the above-mentioned facts, in the current study some observations related to cavitation pitting have been made as follows:-

1. FE computations have shown that the hydrodynamic peak pressure is directly correlated to the pit shape factor and follows a logarithmic law. Moreover, the radial extent of the pressure distribution is virtually proportional to pit diameter defined on the basis of the FCOD method at mid pit depth $(\mathrm{FCOD}=50 \%)$.

2. There exists a common normalized pit shape for all cavitation pits (when pit depth is normalized by maximum depth and corresponding diameter is normalized by maximum diameter).

3. A Gaussian pressure distribution for the applied load appears to be realistic since it leads to a computed mean pit shape close to the measured one.

4. It was also established that a one-to-one correspondence exists between hydrodynamic load parameters $\left(\sigma_{H}, d_{H}\right)$ and pit geometrical parameters $\left(h_{P}, d_{P}\right)$ that makes the proposed inverse technique workable in practice.

Future work is directed towards using the estimated impact loads randomly and repetitively to a material surface to estimate mass loss with time to predict cavitation erosion. For that purpose dynamic explicit FE simulation has to be done considering the strain rate sensitivity of the material along with proper damage model. One difficulty for such method would be in defining the duration of hydrodynamic impact, which cannot be measured experimentally by the current state-of-art. The main results of the dynamic explicit method are presented in Part 2 [20].

\section{Acknowledgements}

This research was conducted under a NICOP project funded by the Office of Naval Research. The authors wish to thank Dr. Ki-Han Kim from the Office of Naval Research (ONR) and Dr. Woei-Min Lin from the Office of Naval Research Global (ONRG) who supported this work. They are also grateful to Michel Riondet (LEGI) for conducting the cavitation pitting tests and Dr. Michel Suery and Mr. Charles Josserond (SIMaP) for the tensile tests. 


\section{References}

[1] C E Brennen, Cavitation and Bubble Dynamics. New York: Oxford University Press, 1995.

[2] C E Brennen, Hydrodynamics of Pumps. Oxford University Press and Concepts, ETI Inc, 1994.

[3] J P Franc and J M Michel, (eds.), Fundamentals of Cavitation. Fluid Mechanics and Its Applications, Vol. 76, Kluwer Academic Publishers, 2004

[4] M S Mihatsch, S J Schmidt, M Thalhamer, and N A Adams, "Quantitative Prediction of Erosion Aggressiveness through Numerical Simulation of 3-D Unsteady Cavitating Flows," in 8th International Symposium on Cavitation, CAV2012, Singapore, 2012.

[5] G L Chahine, "Modeling of Cavitation Dynamics and Interaction with Material," Chapter 6, in K H Kim, G L Chahine, J P Franc and A Karimi. (eds): Advanced Experimental and Numerical Techniques for Cavitation Erosion Prediction. Fluid Mechanics and its Applications, Vol. 160, pp. 123-162, (ed.) Springer, 2014.

[6] S J Schmidt, M S Mihatsch, M Thalhamer, and N A Adams, "Assessment of Erosion Sensitive Areas via Compressible Simulation of Unsteady Cavitating Flows," in Advanced Experimental and Numerical Techniques for Cavitation Erosion Prediction. Springe, 2014.

[7] J P Franc, M Riondet, A Karimi, and G L Chahine, "Impact Load Measurements in an Erosive Cavitating Flow," Journal of Fluids Engineering, vol. 133, no. 12, pp. 121301-1 - 121301-8, 2011.

[8] S Hattori, H Mori, and T Okada, "Quantitative Evaluation of Cavitation Erosion," Journal of Fluids Engineering, vol. 120, no. 1, pp. 179-185, 1998.

[9] H Soyama, A Lichtarowicz, T Momma, and E J Williams, "A New Calibration Method for Dynamically Loaded Transducers and Its Application to Cavitation Impact Measurement," Journal of Fluids Engineering, vol. 120, no. 4, pp. 712-718, 1998.

[10] R T Knapp, "Recent Investigations of the Mechanics of Cavitation and Cavitation Damage," Transactions of the ASME October, vol. 77, pp. 1045-1054, 1955.

[11] R T Knapp, "Accelerated Field Tests of Cavitation Intensity," Transactions of the ASME January, vol. 80, pp. 91-102, 1958.

[12] D Carnelli, A Karimi, and J P Franc, "Application of spherical nanoindentation to determine the pressure of cavitation impacts from pitting tests," Journal of Materials Research, vol. 27, no. 01, pp. 91-99, 2012.

[13] D Carnelli, A Karimi, and J P Franc, "Evaluation of the hydrodynamic pressure of cavitation impacts from stress-strain analysis and geometry of individual pits," Wear, vol. 289, no. 0, pp. 104-111, 2012.

[14] A C Fischer-Cripps, Nanoindentation. New-York, Springer, 2004.

[15] F Pöhl, S Mottyll, R Skoda and S Huth, "Evaluation of cavitation-induced pressure loads applied to material surfaces by finiteelement-assisted pit analysis and numerical investigation of the elasto-plastic deformation of metallic materials", Wear, vol. 330-331, no. 618-628, pp. 1-11, 2015.

[16] G L Chahine, J P Franc and A Karimi, "Cavitation Impulsive Pressures," Chapter 4, in K H Kim, G L Chahine, J P Franc and A Karimi. (eds): Advanced Experimental and Numerical Techniques for Cavitation Erosion Prediction. Fluid Mechanics and its Applications, vol. 160, pp. 71-95, (ed.) Springer, 2014.

[17] S Singh, J -K Choi and G L Chahine, "Characterization of Cavitation Fields from Measured Pressure Signals of Cavitating Jets and Ultrasonic Horns," ASME Journal of Fluids Engineering, vol. 135, no. 9, pp. 091302-1 -091302-11, 2013.

[18] C -T Hsiao, A Jayaprakash, A Kapahi, J -K Choi and G L Chahine, " Modelling of material pitting from cavitation bubble collapse", Journal of Fluid Mechanics (2014), vol. 755, pp. 142-175.

[19] J K Choi, A Jayaprakash, A Kapahi, C T Hsiao and G L Chahine, "Relationship between space and time characteristics of cavitation impact pressures and resulting pits in materials", Journal of Materials Science, vol.49, no. 8, pp. 3034-3051, 2014.

[20] S C Roy, J-P Franc, N. Ranc and M Fivel, "Determination of cavitation load spectra - Part 2: Dynamic finite element approach", submitted to wear, 2015.

[21] S C Roy, J-P Franc and M Fivel, Cavitation erosion: using the target material as a sensor, Submitted to Model. Sim. Mater. Sci. Eng., 2015.

[22] K L Johnson, Contact Mechanics: Cambridge University Press, 1985.

[23] R T Knapp, J W Daily and F G Hammitt, Cavitation. New York: McGraw Hill Book Co, 1970.

[24] Y Tomita and A Shima, "Mechanisms of impulsive pressure generation and damage pit formation by bubble collapse," Journal of Fluid Mechanics, vol. 169, pp. 535-564, 1986.

[25] J Lemaitre and J L Chaboche, Mécanique des matériaux solides. Dunod, 1996.

[26] K Ludwik, "Element der technologischen mechanik," Journal Springer, vol. 32, 1909.

[27] J P Franc, "Incubation time and cavitation erosion rate of work-hardening materials," Journal of Fluids Engineering, vol. 131, no. 2, pp. 021303-1 - 021303-14, 2009.

[28] M Fivel, J -P Franc and S C Roy, "Towards Numerical Prediction of Cavitation Erosion", Interface focus, vol. 5, pp. $20150013-$ 1 - 20150013-10, 2015. 